\title{
CIENCIA\&SALUD
}

\section{ENFERMEDAD INFLAMATORIA INTESTINAL PEDIÁTRICA EN}

COSTA RICA : UNA REVISIÓN DE 15 AÑOS.

\section{PEDIATRIC INFLAMMATORY BOWEL DISEASE IN COSTA RICA:}

\section{A 15 YEARS STUDY}

Gabriela Jiménez Arguedas ${ }^{1}$ Raquel Maria Herrera Rodríguez² Alejandro Víquez Víquez³

1 Médico pediatra gastroenteróloga, Caja costarricense del seguro social San José, Costa Rica.

2 Médico asistente general. Caja costarricense del seguro social San José, Costa Rica.

3 Médico Neonatólogo, Caja costarricense del seguro social San José, Costa Rica.

\section{RESUMEN}

Antecedentes: La enfermedad inflamatoria intestinal (EII) se caracteriza por una in-flamación del intestino secundaria a un proceso autoinmune. En Costa Rica, aún no se tienen datos reales sobre la incidencia y la forma de presentación pediátrica.

Métodos: Estudio retrospectivo, descriptivo y observacional, basado en la revisión de expedientes clínicos. Se tomaron todos los pacientes con diagnóstico de Ell (enfer-medad de Crohn (EC) y colitis ulcerosa (CU) entre los 0 y los 15 años, atendidos en el Hospital pediátrico para el período del 01 de agosto de 2000 al 31 de agosto de 2015.

\section{Cómo citar:}

Jiménez Arguedas, G., Herrera Rodríguez, R., \& Víquez Víquez, A. (2021). ENFERMEDAD INFLAMATORIA INTESTINAL PEDIÁTRICA EN COSTA RICA. Revista Ciencia Y Salud, 5(2), Pág. 95-104.

Recibido: 14/ene/2021 Aceptado: 22/mar/2021 Publicado: 16/abr/2021

Resultados: Se incluyeron 34 pacientes, 30 pacientes con colitis ulcerativa y 4 con enfermedad de Crohn. La tasa de incidencia del estudio fue de 2.96/100000 habitan-tes para edades entre los 0 y los 15 años. La edad promedio al diagnóstico fue 8.8 años. La clínica inicial fue diarrea (85.5\%); en su mayoría sanguinolenta (96.6\%). Los resultados de los laboratorios al diagnóstico fueron: eritrocitos o leucocitos presentes en heces (79.3\%), anemia (57.6\%) y elevación de la VES (50\%). El hallazgo más co-mún en la colonoscopía fue la mucosa friable (88.2\%). Las biopsias en su mayoría fueron inespecíficas y en menos de la mitad de los casos concluyentes de CU o EC. Todos los pacientes se trataron con esteroides. Solo tres pacientes requirieron cirugía. El 58.8\% de los pacientes tuvo recaídas en el primer año del diagnóstico.

Conclusiones: En Costa Rica hay más casos de CU que de EC. La implementación de un protocolo de atención puede lograr un mejor diagnóstico y manejo de pacientes con sospecha de Ell en Costa Rica.

\section{Palabras Clave:}

Enfermedad inflamatoria intestinal, Colitis ulcerosa, enfermedad de Crohn, pediatría.. 


\section{CIENCIA\&SALUD}

\section{ABSTRACT}

Aim: Inflammatory bowel disease (IBD) is a chronic autoimmune inflammation. In Cos-ta Rica, the incidence of pediatric presentation is unknown. The aim of this study is to describe the incidence and clinical characteristics of pediatric IBD in Costa Rica.

Methods: Descriptive retrospective study based on clinical records. All patients diag-nosed with IBD between $\mathrm{O}$ and 15 years of age treated at the National Children's Hos-pital.

Results: Among the 34 patients included, 30 had ulcerative colitis (UC) and 4 Crohns disease (CD). The overall incidence rate was 2.96/100,000. 55.9\% females. The gen-eral average age at diagnosis was 8.8 years. The initial clinical presentation was diar-rhea (85.5\%); mainly dysenteric (96.6\%). Extra intestinal manifestations did not occur in $70.6 \%$. Frequent laboratory findings included erythrocytes and leukocytes in feces (79.3\%), anemia (57.6\%) and elevation of erythrocyte sedimentation rate (50\%). The most common finding on colonoscopy was friable mucosa $(88.2 \%)$. Biopsy results were mostly nonspecific, in less than $50 \%$ conclusive of UC or CD. All patients were treated with steroids. Three patients required surgery. 58.8\% relapsed during the first year after diagnosis, with an average of 1.6 relapses/year.

Conclusion: This is the first pediatric study of its kind in Costa Rica. UC is more frequent than CD. Carrying out a protocol to improve diagnosis and care of patients with IBD is recommended.

\section{Keywords: \\ Antiphospholipid syndrome, massive pulmonary thromboembolism, anticoagulation.}

La enfermedad inflamatoria intestinal es una enfermedad crónica que en muchos ca-sos se presenta en la segunda década. Sus manifestaciones pueden variar desde sín-tomas leves, hasta severos y en algunos casos hasta una presentación fulminante, siendo una enfermedad poco prevalente, tiene implicaciones importantes a nivel so-cioeconómico; pues afecta, de manera muy importante, la forma de vida del paciente, de sus familias y su entorno social.

Establecer la incidencia, epidemiología y forma de presentación de esta enfermedad en la pobalción pediátrica costarricense es necesario para iniciar una base de datos propios, para lograr correlacionar y comparar con los datos que se presentan en otros países, mejorando el diagnóstico y seguimiento de los pacientes. Se realiza un estudio descriptivo, retrospectivo y observacional, cuyo objetivo es deta-llar las características demográficas y clínicas de los pacientes pediátricos con Ell, atendidos en el Hospital pediátrico. 


\section{CIENCIA\&SALUD}

\section{MÉTODOS}

\section{Diseño}

Para obtener la información se utilizó una hoja prediseñada de recolección de datos. Los pacientes fueron identificados según la información consignada por el servicio de documentos médicos y estadística del hospital, atendidos tanto en el servicio de emergencias, consulta externa de gastroenterología y hospitalizados. Este es el único hospital de referencia pediátrico de todo el país.

\section{Criterios de inclusión}

Pacientes entre $\mathrm{O}$ y 15 años con diagnóstico de EII (CU y EC), en el Hospital Pediátri-co, durante el período del 01 de agosto de 2000 al 31 de agosto de 2015 con atención tanto en servicios de emergencia, consulta externa de Gastroenterología y hospitali-zados.

\section{Criterios de exclusión}

Se excluyeron los que tuvieron menos del $40 \%$ de la información requerida para el llenado de la hoja de recolección de datos o que en el expediente no se contara con antecedentes indispensables para el estudio.

\section{Tamaño de la muestra}

La muestra inicial fue de 69 pacientes, de los cuales se excluyeron 28 por presentar un diagnóstico diferente a Ell o no encontrarse el expediente. Además, se descartaron 7 por tener información incompleta. La muestra final fue de 34 pacientes.

\section{Análisis de datos}

Se efectuó un análisis de los datos cualitativos y cuantitativos comúnmente emplea-dos en los estudios clínicos de vigilancia epidemiológica. Para las variables cuantitati-vas se utilizó estadística descriptiva con medidas de tendencia central (media y me-diana) y para las variables cualitativas se realizaron cálculos de frecuencia.

Además, se realizó una tasa de incidencia con base en los datos publicados por el Instituto Nacional de Estadística y Censo de Costa Rica. Se tomó en cuenta la pobla-ción nacional total con edades entre los $\mathrm{O}$ y 15 años y se calculó una tasa por 100.000 habitantes. Los análisis se realizaron usando el software Epidata 3.1 y Microsoft Excel 15.13.1.

\section{Aspectos éticos}

El presente estudio fue aprobado por el Comité Local de Bioética e Investigación del HNN con el código CLOBI-HNN-O21-2014. 


\section{RESULTADOS}

El 55.9\% de los pacientes correspondió al sexo femenino, para una relación hom-bre/mujer de 0.8:1. La edad promedio al momento del diagnóstico fue de 8.8 años. Según el grafico de distribución por provincias, San José tiene una prevalencia del 64.7\%. La tasa de incidencia para este estudio fue de 2.96/100.000 habitantes con edades entre los 0 y los 15 años (Gráfico 1). Otros hallazgos sobre la distribución por edad y sexo se detallan en el cuadro 1.

Gráfico 1. Distribución de pacientes con Enfermedad Inflamatoria Intestinal según provincia de procedencia. Hospital pediátrico, 2000-2015 ( $n=34)$

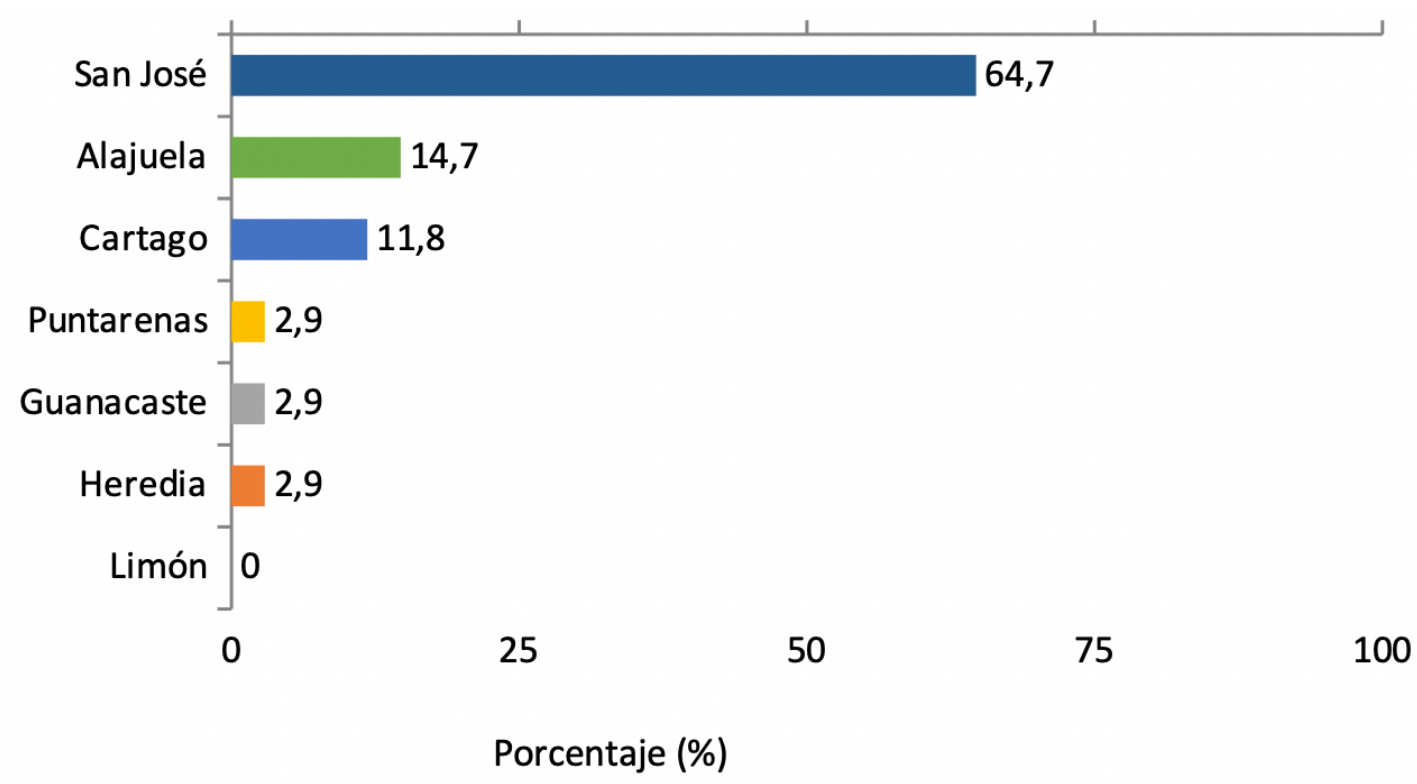

Cuadro 1. Determinación de la edad en años al diagnóstico, en pacientes con Enfermedad Inflamatoria Intestinal según sexo. Hospital pediátrico 2000-2015 ( $n=34)$

\begin{tabular}{|c|c|c|c|c|}
\hline Sexo & $\mathbf{n}$ & $\begin{array}{c}\text { Media } \\
\text { (años) }\end{array}$ & $\begin{array}{c}\text { Mediana } \\
\text { (años) }\end{array}$ & $\begin{array}{c}\text { Rango } \\
\text { (años) }\end{array}$ \\
\hline Masculino & 15 & 8.5 & 9.6 & $2.1-14.1$ \\
\hline Femenino & 19 & 9.1 & 8.6 & $5.0-13.1$ \\
\hline General & 34 & 8.8 & 8.6 & $2.1-14.1$ \\
\hline
\end{tabular}

El 75.8\% de los pacientes requirió hospitalización. La estancia hospitalaria tuvo un tiempo medio de 11.9 días, con un rango de 2-58 días. 


\section{CIENCIA\&SALUD}

La evaluación del estado nutricional inicial documentó que la mayoría de los pacientes estaban eutróficos (67.5\%) (Ver gráfico 2). La presentación clínica evidenció como síntoma más frecuente la diarrea en $85.5 \%$ de casos y de estos en el $96.5 \%$ era sanguinolenta $(n=28 / 29)$. El segundo hallazgo fue el dolor abdominal (70.6\%), se-guido de hematoquecia (55.9\%) y como cuarto el sangrado rectal (35.3\%) (Gráfico 3). La mayoría de pacientes no presentó manifestaciones extraintestinales (70.6\%).

Gráfico 2. Distribución de pacientes con Enfermedad Inflamatoria Intestinal según clasificación nutricional al momento del diagnóstico.

Hospital pediátrico, 2000-2015 $(n=34)$

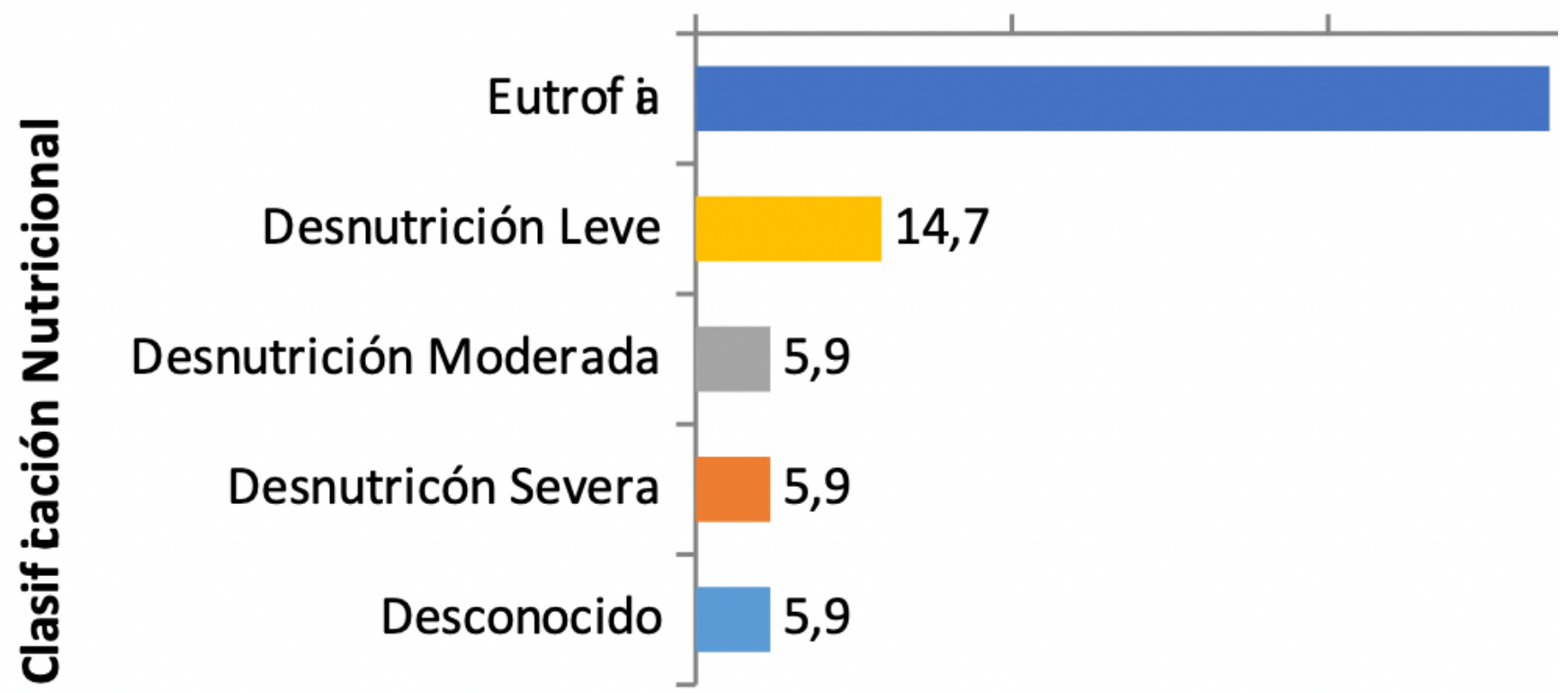

Gráfico 3. Distribución de pacientes con Enfermedad Inflamatoria Intestinal según presentación clínica al diagnóstico. Hospital Nacional de Niños

“Dr. Carlos Sáenz Herrera”, 2000-2015 (n=34)

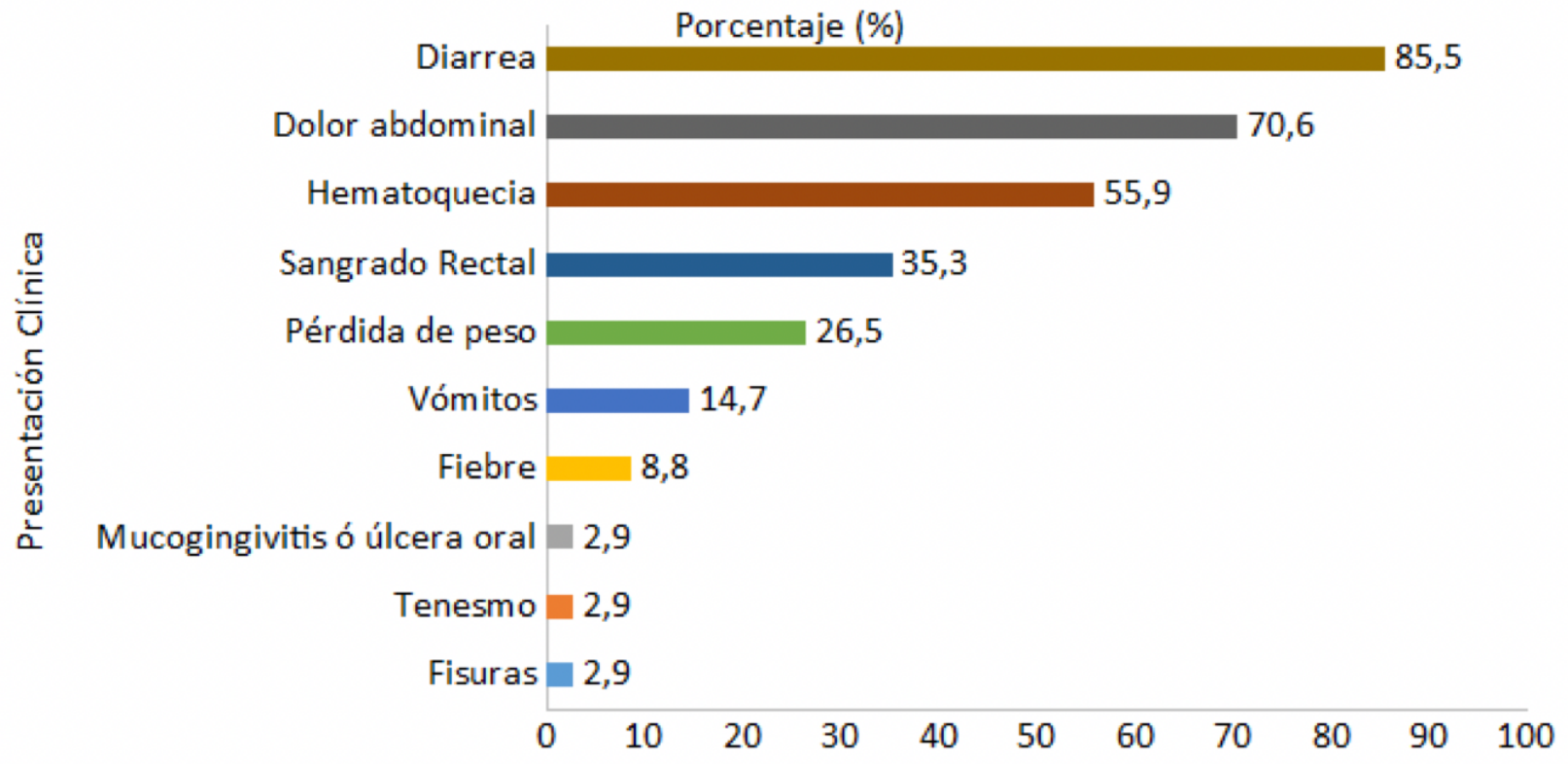




\section{CIENCIA\&SALUD}

Los hallazgos de laboratorio más comunes fueron la presencia de leucocitos y/o eri-trocitos en heces (79.3\%), en segundo lugar, la anemia (57.6\%), seguido de elevación de la VES (50.0\%) y leucocitosis periférica (43.8\%). Hallazgos menos comunes fue-ron la trombocitos periférica (37.5\%), la hipoalbuminemia (24.1\%), la elevaciónn de PCR (20.8\%), la pandemia (15.6\%) y la alteraciónn de las PFH (9.7\%). Se realiza la comparación de estos datos por patología específica en la Figura 1. El látex por Clo-stridium difficile al momento del diagnóstico, en su mayoría, no se realizó (55.9\%).

Figura 1. Hallazgos de laboratorio más frecuentes al diagnóstico, según patología específica: CU y EC. Hospital pediátrico, 2000-2015 $(n=34)$

\section{CU}

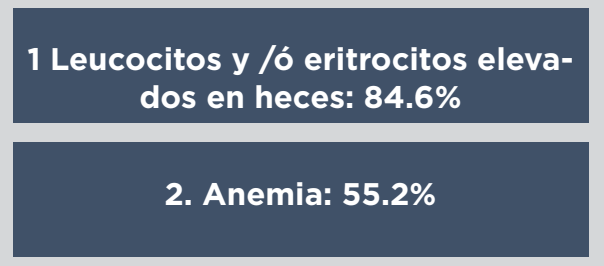

3. Elevación: VES $42.9 \%$

4. Leucocitosis: $\mathbf{3 5 . 7} \%$

\section{EC}

1 Leucocitosis: $100.0 \%$

2. Trombocitosis: $100.0 \%$

3. Anemia: $75.0 \%$

4. Elevación VES: $75.0 \%$

Dentro de los estudios endoscópicos, se evidenció que la gastroscopía no fue realiza-da en $44.1 \%$ de los casos, un $41.2 \%$ fue descrita como normal y en un $14.7 \%$ fue anormal. El principal hallazgo microscópico fue la mucosa edematosa y eritematosa.

A todos los pacientes se les realizó colonoscopía $(n=34)$. La alteración más común fue la mucosa friable o eritematosa (88.2\%), seguida de edema e inflamación (79.4\%). Además, se evidenciaron úlceras o microulceraciones en el 70.6\%. Cambios menos frecuentes fueron la presencia de pseudopólipos (17.6\%), la pérdida de la arquitectu-ra y del patrón vascular (14.7\%), patrón granular y erosiones (5.9\%) y lesiones en sa-cabocados, sangrado espontáneo y patrón en parche (2.9\%). Al final se documentó que el 100\% presentaba afección colónica, $82.4 \%$ rectal, $29.4 \%$ perianal y $11.8 \%$ en ileon.

Finalmente, según el tipo de Ell se clasificó a un $88.2 \%$ como CU y a un 11.8\% EC. La figura 2 muestra las alteraciones colonoscópicas más relevantes según la patología específica. 
Figura 2. Hallazgos macroscópicos de colonoscopía más frecuentes según pato-logía específica: CU y EC. Hospital pediatrico, 2000-2015 $(n=34)$

\section{CU}

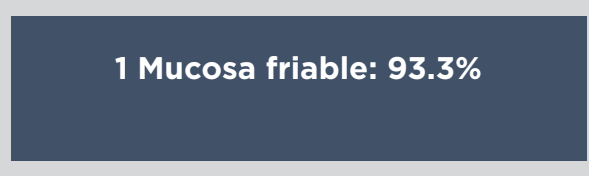

2. Inflamación: $\mathbf{8 0 . 0 \%}$

3. Úlceras $66.6 \%$

4. Pérdida arquitectura: $20.0 \%$

${ }^{*}$ Erosiones6.6\%

* Sangrado espontàneo: $3.3 \%$

\section{EC}

\section{1 Úlceras: $100.0 \%$}

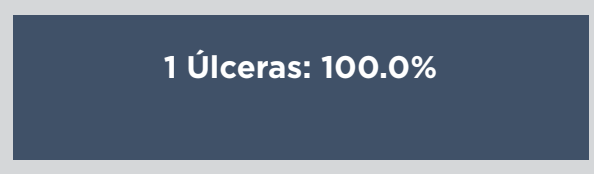

2. Inflamación: $\mathbf{1 0 0 . 0 \%}$

3. Mucosa friable: $\mathbf{5 0 . 0 \%}$

4. Pseudopólipos: $\mathbf{5 0 . 0 \%}$

\section{* Aftas: $\mathbf{2 5 . 0 \%}$}

* Patrón parche: $\mathbf{2 5 . 0 \%}$

* Hallazgo único en la patología

Los resultados histológicos mostraron que el $44.1 \%$ de los pacientes presentaba CU, el 20.6\% hiperplasia linfoide o inespecífica inflamatoria, un 14.7\% Ell crónica y un 5.9\% Ell idiopática. Solo un 2.9\% de los casos se reportaron como enfermedad de Crohn (Gráfico 4).

En el 100\% de los pacientes se utilizó glucocorticoides como tratamiento para lograr la remisión de los síntomas, con una duración promedio de 13.5 semanas y un rango de 4 a 54 semanas.

La azatioprina se utilizó para la remisión en un $17.2 \%(n=5 / 29)$, mantenimiento $31.0 \%(n=9 / 29)$ y para ambas etapas de tratamiento en $51.7 \%(n=15 / 29)$.

La 5-ASA se utilizó de la siguiente forma: en mantenimiento $(50 \%, n=6 / 12)$, para la remisión en un $8.3 \%(n=$ $1 / 12$ ) y en las dos fases del tratamiento en el $41.6 \%$ de los pacientes $(n=5 / 12)$. 


\section{CIENCIA\&SALUD}

Gráfico 4. Distribución de pacientes con Enfermedad Inflamatoria Intestinal según resultado de la biopsia. Hospital pediátrico, 2000-2015 $(n=34)$

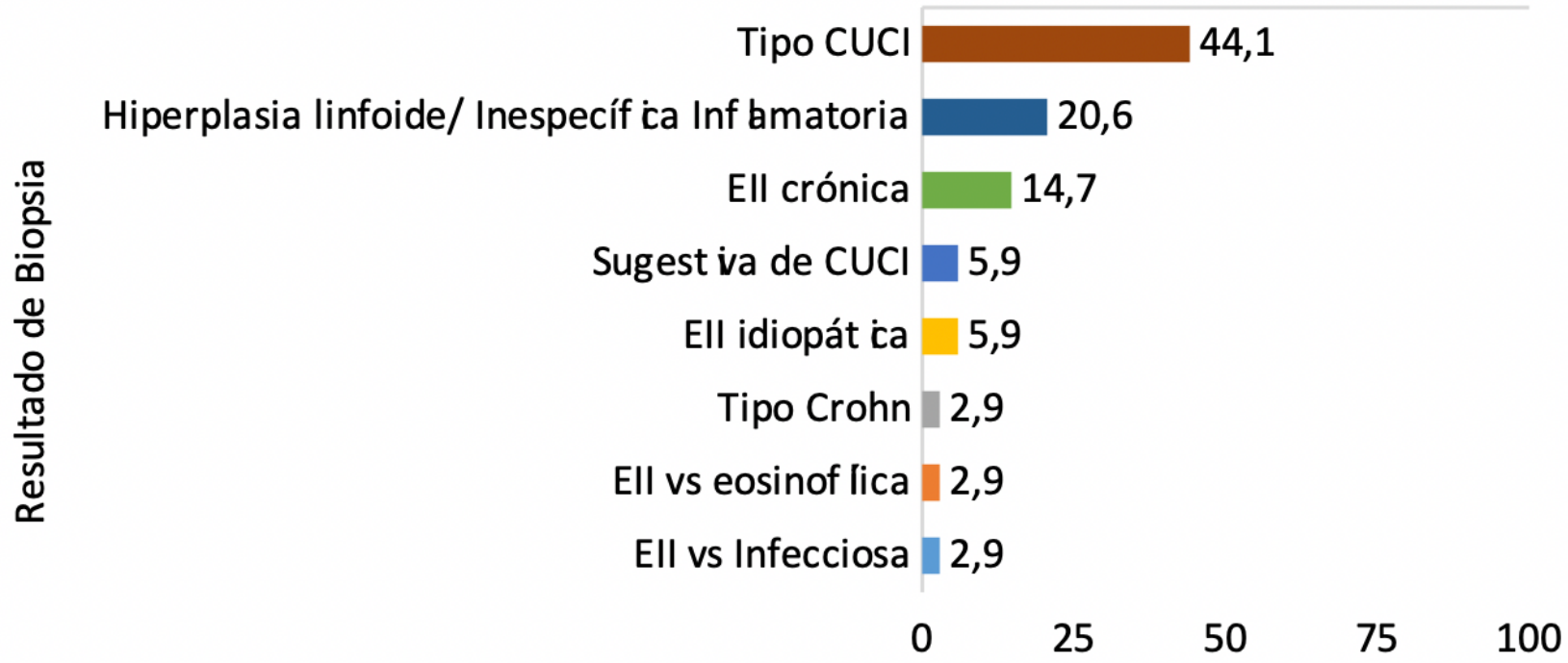

La cirugía fue necesaria en tres pacientes para un 8.8\% de la muestra. Dos de ellos requirieron colectomía total, anastomosis ileo-anal e ileostomía. El último paciente tenía EC y ameritó resección de la válvula ileocecal más anastomosis término-terminal.

Entre las complicaciones documentadas están las recaídas al año de establecido el diagnóstico, en un 58.8\% $(n=20 / 34)$ con una media de 1.6 recaídas/año y un rango de 1 a 3 . Pacientes esteroide dependendientes en un $17.6 \%(n=6 / 34)$. Por otro lado, se documentó un paciente con $\mathrm{CU}$, úlcera sacra, estenosis rectal post cirugía y obstruc-ción intestinal por bridas. También un caso con CU presentó transfusiones múltiples y toxicidad medular por azatioprina. Por último, un niño con EC tuvo un absceso rectal y una fístula perianal.

\section{DISCUSIÓN}

La distribucion según sexo no demostró ningún sexo predominante, siendo esto acor-de con la literatura internacional $(1,2,3,4)$.

La edad al diagnóstico reportada en publicaciones es de 9 años (rango de 10 a 15 años) (2,5,6). Al correlacionar esta revisión con nuestros datos, se evidenció una edad de presentación promedio de 8.8 años, para los pacientes.

La tasa de 2.96/100.000 habitantes con edades entre los 0 y los 15 años se decidió establecerla para el estudio y no formalizarla para todo el país. Lo anterior porque po-drían existir otros casos en niños mayores de 13 años que se diagnostican y se tratan en otros hospitales (7). Esta incidencia es comparable con la reportada para la mayo-ría de países europeos nórdicos y meridionales, pero menor que lo reportado para Estados Unidos, Australia y Nueva Zelanda $(1,8)$.

La presentación clínica de la Ell es variable; puede ser un único síntoma inespecífico o diversas molestias características de la enfermedad. En esta población, la queja más frecuente fue la diarrea sanguinolenta, 


\section{CIENCIA\&SALUD}

acompañada de dolor abdominal. Las fi-suras y el tenesmo fueron los menos reportados. Esto último difiere con lo reportado en la literatura, y podría deberse a la carencia del clínico en la búsqueda del tenesmo en la historia clínica $(1,8,9)$.

Estos hallazgos son los esperados al revisar lo reportado mundialmente, donde se espera que un 25-30\% de pacientes las presenten, traduciendo patología inflamatoria de otros órganos y tejidos diferentes al TGI $(1,4,8)$.

Los hallazgos de laboratorios encontrados son compatibles con inflamación intestinal crónica. La hipoalbuminemia y trombocitosis no fueron tan frecuentes, como en otros estudios. Es importante tomar en cuenta que no se encontró ningún caso positivo con $\mathrm{C}$. difficile, ya que esta es una prueba que en la institución se realiza hasta hace poco tiempo; la literatura reporta casos diagnosticados en relación y posterior a una infec-ción por este gérmen $(1,8,9)$.

El análisis de las endoscopías mostró que no se realiza una gastroscopía en todos los pacientes con sospecha de Ell. Este estudio debe ser mandatorio en el tamizaje diag-nóstico.

La descripción de los cambios macroscópicos debería quedar más detallada en los expedientes clínicos, para así mejorar el diagnóstico diferencial entre CU o EC. Las áreas con mayor afectación fueron el colon, recto y región perianal, lo que es compa-rable y esperable según lo descrito para la patología $(1,8)$.

Con respecto a las biopsias, lo descrito en su mayoría es inespecífico para una ade-cuada clasificación de la enfermedad como CU o EC. Por lo tanto, no se puede clasifi-car una enfermedad específica ni se descarta la posibilidad de causas como las infec-ciosas. Esto es una limitante importante para el clínico.

La clasificación final del paciente se debe hacer tomando en cuenta: la clínica, labora-torios, hallazgos endoscópicos e histológicos; mostró que en esta población la mayo-ría de casos correspondieron a CU. Esto difiere con la mayoría de países donde se describe una mayor incidencia de EC. Es importante tomar en cuenta que, para países en vías de desarrollo como Costa Rica, la literatura menciona que la CU podría ser más común $(1,8)$.

Todos los niños de este estudio fueron tratados con esteroides. En promedio, la remi-sión se logró a las 13.5 semanas; período un poco mayor a lo contemplado en los tex-tos. Se usaron las dos fases de tratamiento; remisión y mantenimiento. Los medica-mentos se utilizaron de forma adecuada en cada fase según lo recomendado en la literatura. El uso de tratamiento específico denominado tratamiento Biológico, no se había implementado al momento de realizar esta revisión de expedientes $(1,10,11,12)$.

La mayoría de los niños tuvo al menos una recaída durante el primer año desde el diagnóstico. Solo tres de los pacientes requirieron cirugías; a nivel mundial un $70-75 \%$ de los casos de EC y de $25-30 \%$ en CU deben ser operados $(1,2,6,9,10,11)$.

Esta revisión sobre Ell es la primera que se realiza en niños en Costa Rica. Tiene to-das las limitaciones de un estudio retrospectivo, es el inicio para lograr un manejo in-terdisciplinario y protocolizado de estos pacientes. 


\section{CIENCIA\&SSALUD}

\section{REFERENCIAS BIBLIOGRÁFICAS}

1.Bernstein, C. et al. World Gastroenterology Organization Global Guidelines: In-flammatory Bowel Disease. World Gastroenterology Organization. 2015. Dis-ponible en: www.worldgastroenterology.org 2.Turunen, P. Kolho, K. Auvinen, A. et al. Incidence of inflammatory bowel disease in finnish children, 1987-2003. IBD Journal. 2006; 12(8): 677-683

3.Malaty, H. Fan X, Openkun, A. et al. Rising incidence of Inflammatory Bowel Dis-ease among children: A 12-year study. J Pediatr Gastroenterol Nutr. 2010; 50(1): 27-31.

4.Bousvaros, A. Burpee, T. Leichtner, A. Clinical manifestations of Crohn's disease in children and adolescents. UpToDate. (Última revisión Ene, 2013)

5.Abramson, O. Durant, M. Mow, W. et al. Incidence, prevalence, and time trends of pediatric Inflammatory Bowel Disease in Northern California, 1996 to 2006. J Pediatr. 2010; 157(2): 233-239

6.Adamiak, T. Walkiewicz-Jedrzejczak, D. Fish, D. et al. Incidence, Clinical Char-acteristics, and Natural History of Pediatric IBD in Wisconsin: a Population-based Epidemiological Study. Inflamm Bowel Dis. 2013; 19(6): 1218-1223.

7.Instituto Nacional de Estadística y Censos de Costa Rica. X Censo Nacional de Población y VI Censo Nacional de Vivienda. Archivo: C02. Población total, zo-na, sexo, provincia, edad simple (1).xls. 2011. Disponible en: http://www.inec.go.cr/

8.IBD Working Group of the European Society for Paediatric Gastroenterology, Hepatology and Nutrition. Inflammatory bowel disease in children and adoles-cents: recommendations for diagnosis--the Porto criteria. J Pediatr Gastroenterol Nutr. 2005;41(1):1-7. doi:10.1097/01.mpg.0000163736.30261.82 1.Ponsky, T. Hindle, A. Sandler, A. MD. Inflammatory Bowel Disease in the pediat-ric patient. Surg Clin North Am. 2007; 87(3): 643-658

9. Peppercorn, M. Farrell, R. Management of severe ulcerative colitis. UpToDate. (Última revisión Jun, 2014).

10.Bousvaros, A. Leichtner, A. Overview of the management of Crohn's disease in children and adolescents. UpToDate. (Última revisión Mar, 2014)

11.Sauer, C. Kugathasan, S. Pediatric Inflammatory Bowel Disease: Highlighting pediatric differences in IBD. Med Clin North Am. 2010; 94(1): 35-52 